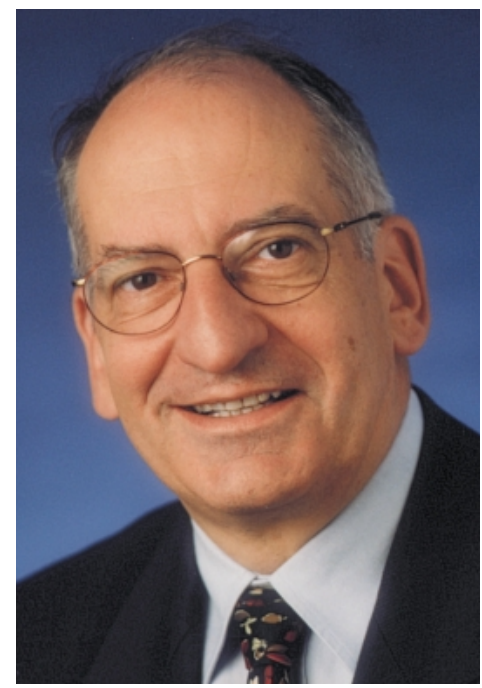

\title{
Accords bilatéraux: les médecins suisses ne sont pas menacés
}

\author{
Pascal Couchepin, conseiller fédéral, chef du DFE
}

On ne saurait trop souligner l'importance des accords bilatéraux pour la Suisse. Près de dix ans après l'instauration du marché intérieur européen et le non de la Suisse à l'EEE, ces accords offrent quasiment à notre pays le libre accès à ce marché essentiel pour notre économie. L'importance des accords est aussi psychologique: plus d'un quart de siècle après la conclusion de l'accord de libre-échange avec la Communauté économique européenne (1972), il est temps que nous fassions un pas en direction de la normalisation de nos rapports avec l'UE, même si ce pas est modeste au regard des progrès accomplis dans le processus d'unification de l'Europe. Avec les accords bilatéraux, nous ne comblons certes pas le fossé qui nous sépare du marché intérieur, mais nous édifions plusieurs ponts qui facilitent considérablement l'accès à ce marché pour les personnes, les biens et les services.

L'un des accords les plus importants est sans doute l'accord sur la libre circulation des personnes. Il permet aux entreprises suisses de subvenir à leurs besoins de personnel qualifié - ce dont toute l'économie profitera - et il offre en particulier à nos jeunes concitoyens et concitoyennes la possibilité d'acquérir une expérience professionnelle à l'étranger. Il constitue une solution taillée sur mesure en fonction des besoins et des réserves de la Suisse, solution qui permet une ouverture prudente assortie de nombreuses garanties.

Dans les négociations, c'est toujours donnantdonnant. Naturellement, la Suisse a dû accepter des compromis dans l'intérêt général. Les accords forment ensemble un paquet qui ne peut être mis en vigueur ou abrogé que globalement. Il n'est pas possible de ne prendre que les aspects positifs, par exemple l'accord - très important pour nos compagnies aériennes - sur la libéralisation du trafic aérien ou celui - ardemment souhaité par l'industrie pharmaceutique et des machines - sur l'abolition des entraves techniques au commerce. Mais le Conseil fédéral est convaincu que le bilan global est nettement positif.
Cette ouverture du marché est fondamentalement positive également pour les médecins et le personnel médical. Elle offre des possibilités aux médecins suisses dans l'UE et peut apporter à la Suisse un meilleur approvisionnement en services médicaux, notamment dans certains domaines de la médecine spécialisée.

Je sais que cette ouverture prête à discussion au sein du corps médical et je comprends les craintes des médecins suisses. Pourtant, les accords ne constituent nullement une menace pour les médecins suisses ni pour aucune autre profession.

La forte densité des médecins en Suisse et les importants investissements que nécessite l'ouverture d'un cabinet médical font qu'il ne faut pas s'attendre à un afflux massif de médecins venant d'Allemagne, d'Autriche, de France ou d'Italie. Il est certes possible que parmi les quelque 2300 médecins provenant de l'UE qui travaillent dans les hôpitaux suisses (ce qui représente environ 8 pour cent du corps médical en Suisse), quelques-uns profitent des nouvelles possibilités professionnelles. Il se peut donc qu'il y ait passagèrement une augmentation du nombre des ouvertures de cabinets médicaux. Mais il ne s'agit que d'un problème transitoire qui ne devrait pas avoir d'effets sensibles sur l'évolution à long terme du nombre des médecins. Cela est confirmé par les expériences réalisées dans l'UE, en particulier en Autriche. Si, contre toute attente, le nombre des médecins établis devait fortement augmenter, le Conseil fédéral dispose des instruments nécessaires pour limiter l'accès aux activités prises en charge par l'assurance obligatoire des soins médicaux et pharmaceutiques (arrêté du Parlement, mars 2000). Mais je suis convaincu que l'excellente qualité des prestations des médecins suisses et la confiance des patients - que nos médecins méritent - seront suffisantes pour faire face à un éventuel renforcement de la concurrence. 